\title{
Visual and somesthetic influences on postural orientation in the median plane
}

\author{
KENNETH NEMIRE and MALCOLM M. COHEN \\ NASA-Ames Research Center, Moffett Field, California
}

\begin{abstract}
We investigated optic and somesthetic contributions to perceived body orientation in the pitch dimension. In a within-subject factorial design, each of 12 subjects attempted to set his/her body erect or $45^{\circ}$ back from erect while restrained in a movable bed surrounded by an adjustable box. The box provided a visual environment consisting of either a grid pattern, two luminous lines, or complete darkness. Both the grid pattern and the luminous lines were effective at biasing settings of body position when the box was pitched; the pitched grid was more effective than the pitched lines. Although the pitch of the box influenced orientation to both goals, the effect was greater for the diagonal goal than for the erect goal. We present a model of postural orientation in the median plane that involves vestibular, somatosensory, and visual inputs.
\end{abstract}

Many models of human spatial perception and orientation are based on data obtained from investigations of the ability of subjects to adjust a line to the visual vertical while the body is stationary (Bischoff, 1974; Mittelstaedt, 1985, 1986; Schone \& Udo de Haes, 1971; Young, 1984). Although setting a line to the vertical is nominally one of exocentric orientation (Howard, 1982) because both the line and the vertical reference are external to the subject, the task is known to be influenced by the static orientation of the body. This influence is generally discussed with respect to transformations of the vestibular signal (Bischoff, 1974; Mittelstaedt, 1985, 1986; Schone \& Udo de Haes, 1971; Young, 1984); other sources of information about body position in these papers are generally ignored. It is important to study static orientation of the body to obtain a complete understanding of spatial perception and orientation.

There are few studies of the influences of stationary visual environments on spatial perception and postural orientation with respect to gravity, a "semiegocentric" orientation task (Howard, 1982). While the perception of visual vertical may depend on the orientation of the body, the perception of body orientation can logically depend solely on vestibular, proprioceptive, ${ }^{1}$ and cutaneous cues; visual information about orientation is not necessarily required. In the present study, we investigate the influence of visual and nonvisual cues on the perception of static

This work was supported by a National Research Council/NASA-Ames Research Center Fellowship to K. N. and by NASA Grant UPN 19916-12-08 to M. M. C. We thank Robert B. Welch, Arnold Stoper, Dominic Massaro, Sheldon Ebenholtz, Bruce Bridgeman, and two anonymous reviewers for thoughtful comments on earlier drafts of this manuscript and Dan Gundo, Alan Hsu, Charles DeRoshia, and Steve McKnight of Graphic Services, Anne Wilson of Publication Services, and Michael Spivey, Annabel Rorden, and Jill Cohen for technical assistance. We also appreciate the help of each of the students who volunteered as subjects for this experiment. Correspondence should be directed to K. Nemire, NASA-Ames Research Center, Mail Stop 262-2, Moffett Field, CA 94035-1000 (e-mail: nemire@eos.arc.nasa.gov). postural orientation. Previous studies of static postural orientation have been concerned with orientation in the roll dimension ${ }^{2}$ or specific manipulation of only one or two sensory cues. We extend this research by employing a multifactorial design and studying static postural orientation in the pitch dimension. We are not currently concerned with dynamic situations of moving stimuli or of postural sway (Andersen \& Braunstein, 1985; Lee \& Aronson, 1974; Nashner, 1971; Stoffregen, 1986; Young, Oman, \& Dichgans, 1975), so we will not further discuss that literature.

\section{Spatial Orientation in the Dark}

In one method of studying postural orientation, a subject, in darkness, is initially displaced from the vertical (erect) position and is asked to return to that erect position; mean errors are generally in the direction of the initial tilt (Howard \& Templeton, 1966). Data from both labyrinthine-defective and labyrinthine-intact subjects suggest that the errors after prolonged body tilt are due to adaptation of somesthetic (proprioceptive and cutaneous) cues (Clark \& Graybiel, 1963, 1964). We do not know whether adaptation of these same cues has similar effects on orientation in illuminated environments.

\section{Spatial Orientation in Illuminated Environments}

Effects of visual stimuli: Orientation of the optic array. A tilted visual frame of reference causes errors in postural orientation (Howard, 1982; Howard \& Templeton, 1966; Witkin, 1949). Orientation errors are generally in the direction of frame tilt when the frame is tilted laterally (rolled) from the vertical (Ebenholtz \& Benzschawel, 1977; Passey, 1950; Sigman, Goodenough, \& Flannagan, 1979). Preliminary data collected in our laboratory suggest that similar effects may be obtained for visual frames that are deviated from vertical about the pitch axis (Emmert, 1989). We are interested in how the effects of a deviated visual frame of reference combine 
with the effects of somesthetic stimulation to influence the perception of postural orientation in the median plane.

Effects of visual stimuli: Structure of the optic array. The pattern and organization of elements in an optic array (Gibson, 1961) define its structure, but the precise characteristics of a tilted array that are responsible for influencing spatial orientation are not well understood. For example, Asch and Witkin (1948a, 1948b) asked subjects to align a rod with the visual vertical while viewing two different tilted scenes. Results indicated that viewing a tilted room biased settings of the rod more than did viewing a tilted luminous frame, but because the room differed from the frame along many dimensions, such as spatial extent, spatial frequency, area of retinal stimulation, and presence of objects with polar features (such as a chair and a table), it is not possible to determine which aspects of the room were most responsible for its influence on spatial orientation.

Subsequent investigations of spatial orientation have defined some of the parameters of tilted luminous frames that are influential in biasing orientation. Increased retinal size of a tilted frame results in larger deviations in adjusting a rod, or in setting one's head to the apparent vertical (Ebenholtz, 1977; Ebenholtz \& Benzschawel, 1977), and complete frames are more effective than partial frames in biasing spatial orientation (Streibel, Barnes, Julness, \& Ebenholtz, 1980).

Other investigations of spatial orientation in altered visual environments have failed to find additional biasing influences of enhanced structure. Singer, Purcell, and Austin (1970) found that although a tilted white room biased subjects' visual alignment of a rod to the vertical, neither the addition of polar objects nor the addition of stripes to the back wall of the room resulted in larger biases. Similarly, Matin, Fox, and Doktorsky (1987) found that the biasing effect of two pitched parallel luminous lines on visually perceived eye level was not significantly different from that resulting from a pitched richly structured environment (Matin \& Fox, 1989). Subsequent experiments in their laboratory suggest that a single pitched line is sufficient to bias spatial perception (Matin \& Li, $1989,1992)$ and have led to a model of visual spatial perception (Matin \& Li, 1992). We examine whether such minimal optic structure of the pitched array is sufficient to bias postural orientation in the pitch dimension. The results should be important for a more general model of visual spatial orientation that includes postural orientation.

To begin our investigation of the characteristics of the optic array responsible for the effects of pitched environments on postural orientation, we chose two visual stimuli that represent different amounts of optic structure: a pair of parallel lines (after Matin et al., 1987) and a grid pattern. It is possible that visual cues are more informative for judgments of eye level than for judgments of body position; we examine the influence of pitched optic arrays on the perception of body position.

\section{Relative Contributions of Somesthetic and Visual Information}

Interactions of optic and somesthetic stimuli. In the studies cited, tilted optic arrays biased the perception of the visual vertical and the perception of body orientation, despite the presence of veridical vestibular, proprioceptive, and cutaneous cues to spatial orientation. We are interested in how visual and somesthetic cues interact to influence the perception of body orientation with respect to gravity. Investigators in several laboratories reported a smaller bias of tilted visual environments on adjusting a rod to the vertical when a subject is erect than when not erect (e.g., Goodenough, Oltman, Sigman, \& Cox, 1981; Lechner-Steinleitner \& Schone, 1980; Lichtenstein \& Saucer, 1974; Templeton, 1973). They argue that vestibular cues are more accurate and less variable when the body is erect than when it is not (Knight, 1958; Nelson, $1967,1968)$. As a result, they suggest that the subject relies on vestibular information more strongly in the erect position, and thus the optic bias of the tilted visual frame is reduced. Some of these investigators also note that other, nonvestibular, changes in sensory stimulation may contribute to the observed biases. It is possible that enhanced proprioceptive and cutaneous cues in the upright position are responsible for the diminished influence of visual stimuli on spatial orientation when the body is erect. For example, Nyborg (1971) found that a tilted visual frame biased spatial orientation (as indicated by adjusting a rod to the vertical) less when the tilted body was supported by a rigid structure than when it was supported by a padded structure.

Visual capture and bias ratios. We refer to the influence of a tilted or pitched optic array on spatial orientation as "optic bias" (Stoper \& Cohen, 1989). The magnitude of this effect can be expressed as an "optic bias ratio," defined here as the ratio of the actual measured bias (i.e., the deviation of body orientation from the attempted goal) to the total deviation (i.e., the angle) of the pitched optic array. Consider an example in which the subject attempts to orient the body to the vertical position while viewing an optic array that is pitched $20^{\circ}$ forward (or down). If the body was set $10^{\circ}$ forward from the vertical, the resultant optic bias ratio would be $10^{\circ} / 20^{\circ}$, or $50 \%$. A bias ratio of $0 \%$ would indicate that the array has no influence on body orientation, whereas a ratio of $100 \%$ would indicate that the optic array completely determines perceived body orientation. Thus, bias ratios between $0 \%$ and $100 \%$ indicate the relative contributions of visual and nonvisual inputs as they determine perceived body orientation.

In using the bias ratio, we assume that subjects can accurately estimate postural orientation and the angle of a pitched optic surface under conditions in which somesthetic and visual cues are not in conflict. This assumption is not an issue in the current experiment, however, because we compare the effects obtained in each of our 
experimental conditions with effects obtained in our control conditions.

\section{Rationale for the Current Experiment}

We believe that the understanding of optic-somesthetic influences on spatial orientation may be furthered by investigating the perception of the orientation of the body as well as the perception of the orientation of visual objects, body orientation in the pitch as well as the roll dimension, and body orientation in static as well as dynamic situations. Our multifactorial design allows quantification of the interactions between several sources of information that may influence spatial orientation; this is not possible with examination of the influences of single sensory modalities.

Our primary objective is to determine the influence of both the pitch and the structure of an optic array on perception and orientation of the body with respect to gravity. Our independent variables are box pitch, optic structure, initial bed pitch, and goal orientation. If pitching an optic array that surrounds the subject (a box) biases body orientation to a gravitational reference in the same direction as the direction in which the box is pitched, we can quantify the influence of optic pitch on postural orientation. Varying the optic structure of the box enables us to determine the extent to which the influence of the pitched array depends on its pattern. Manipulation of starting position (initial bed pitch) by changing the initial distribution of forces on the body provides an opportunity to study the influence of adaptation of somesthetic cues on postural orientation. The variable of goal orientation allows us to investigate how the influence of visual cues on postural orientation changes as a function of the different patterns of vestibular and somesthetic information that are available in the two orientations.

The amount of optic bias or "visual capture" (Hay, Pick, \& Ikeda, 1965; Matin, 1986) may depend on the relative precision of the sensory modalities involved; a perceptual modality that is more precise (e.g., vision) will partially "capture" a modality that is less precise (e.g., proprioception; for reviews, see Welch \& Warren, 1980, 1986). In the case of the present study, we expect that the amount of optic bias of body orientation resulting from a pitched visual array will depend on the variability in perceived body position; the greater the variability, the greater will be the optic bias.

\section{METHOD}

\section{Subjects}

Twelve undergraduate students, 6 females and 6 males, volunteered as subjects, in partial fulfillment of requirements for a psychology course at a nearby university. Ages ranged from 18 to 28 years, with a mean of 21.3 years. All subjects had normal or corrected-to-normal vision and had no obvious vestibular or motor dysfunction. None of the subjects were aware of our experimental hypotheses.

\section{Apparatus}

The subjects were restrained, face up, in a motorized Stryker CircOlectric hospital bed (Figure 1) which could rotate in the pitch dimension. The angle of bed pitch was registered by a clinometer that could be read to an accuracy of $0.5^{\circ}$. Head position was maintained by a Bausch and Lomb padded head restraint attached to the bed frame. Body position was constrained by means of the standard equipment supplied with the bed. Foam padding, $5 \mathrm{~cm}$ thick, cushioned the dorsal and ventral aspects of the subject's body. Additional foam padding surrounded the subject's arms to prevent arm movement. The foam padding between the subject's feet and the foot support of the bed frame was $10 \mathrm{~cm}$ thick. The subject and the experimenter controlled the position of the bed with separate hand-held toggle switches. The speed of bed rotation was $6^{\circ}$ per second, which was experienced as a fairly slow and pleasant, if unusual, ride.

The bed was surrounded by a rectangular aluminum frame with foam-core walls. The frame, $122 \mathrm{~cm}$ wide $\times 168 \mathrm{~cm}$ high $\times$ $279 \mathrm{~cm}$ long, was supported by a stand that acted as the fulcrum for rotation about the midpoint of its longer walls.

Inside the box, the subject was either in total darkness or was presented with one of two types of visual stimuli: two parallel luminous lines or a grid pattern. The luminous lines consisted of two electroluminescent panels, each $0.9 \mathrm{~cm}$ wide $\times 134.6 \mathrm{~cm}$ long (Figure 2). The lines were placed on the outer edges of the wall facing the subject. When the box was level, the lines were vertical with respect to gravity. Luminance was approximately $0.009 \mathrm{~mL}$.

The grid pattern, provided by wallpaper completely covering the inside of each surface of the box (Figure 2), consisted of $5.2 \mathrm{~cm}$ $\times 5.2 \mathrm{~cm}$ squares that each contained a grid of 64 smaller squares; the grid comprised dark blue lines on a white background. Illumination inside the box was provided by two fluorescent lamps ( $15 \mathrm{~W}$ ) attached to the body restraint; the fixed position provided, as much as possible, equivalent illumination for all experimental conditions. The distance from the subject's eyes to the top and front walls of the box varied as a function of bed and box position. The retinal angle subtended by the horizontal crossbar (Figure 2, Line Segment $\mathrm{AC}$ ) varied from $27^{\circ}$ to $36^{\circ}$ for the diagonal positions and from $35^{\circ}$ to $54^{\circ}$ for the vertical positions of the subject's body.

Black cloth covering the floor and suspended from the bottom of the frame of the box prevented enclosed subjects from viewing

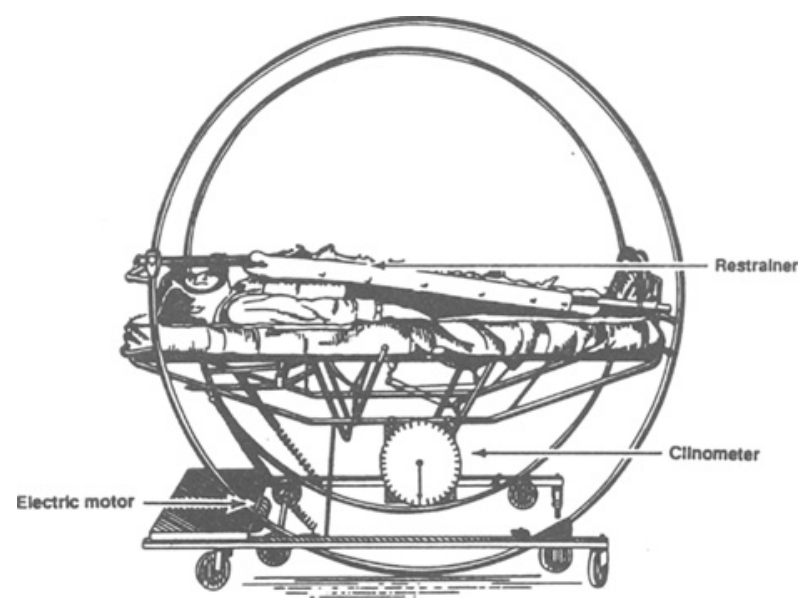

Figure 1. Stryker CircOlectric bed used to position the subjects in the median plane. The bed is driven by an electric motor that is controlled by a hand-held toggle switch (after Cohen \& Larson, 1974). 


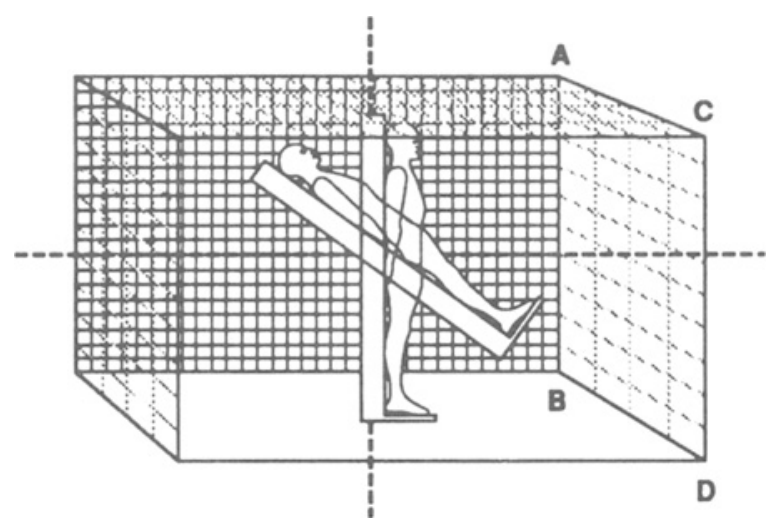

Figure 2. View of the bed and surrounding box; subject at erect and diagonal goals with the pitch box level. The grid pattern, represented here on only one wall, was actually on all inside walls of the box. Line Segments $\mathrm{AB}$ and $\mathrm{CD}$ indicate the location of the parallel luminous lines.

the external room. A fan attached to the bottom of the bed frame ventilated the enclosed area of the box. During data-acquisition trials, the experimenter communicated with the subject via an FM transceiver in a headset to preclude auditory orientation cues.

\section{Procedure}

The subjects read, acknowledged understanding of, and signed a list of instructions and an informed consent. The experimenter answered procedural questions and provided oral instructions and visual aids to further illustrate task requirements. The experimenter then restrained each subject in the bed, gave instructions in the use of the electric controls, and adjusted the headphones. Experimental trials were begun only after the subject had practiced with the controls, become proficient, and indicated that he/she was comfortable.

The task for each subject was to position the body at one of two goal orientations: erect or $45^{\circ}$ back from erect. The subjects were told that they were to orient themselves with respect to gravity and not with respect to the frame of reference provided by the box. These instructions were provided in written and oral form and were further elaborated in drawings. The experimenter also demonstrated the independent movement of the bed and the surrounding box; the subjects were informed that the apparent position of the box did not necessarily indicate the gravitational reference frame.

Three levels of optic structure were presented inside the box: a grid pattern, a pair of parallel luminous lines, or complete darkness. The grid stimulus was presented by illuminating the interior of the box; the external room was also illuminated. The procedure for each trial was as follows. After asking the subject to close his/her eyes, the experimenter moved the box and the bed to the starting positions; the bed was rotated fore and aft of the initial position at least four times to control for unwanted biasing effects of labyrinthine stimulation. The subject was then asked to open his/her eyes and to remain motionless. After a 10-sec delay, the subject was asked to move to the selected goal. After the subject signaled achievement of the goal, the experimenter recorded the angle of bed orientation.

For the luminous parallel line condition, box and room lights were extinguished and power was supplied to the electroluminescent panels. Before proceeding with data-collection trials, the experimenter determined that the subject could clearly see the luminous strips. The procedure for each trial was the same as for the grid condition.

For the dark condition, a blindfold was placed over the subject's closed eyes; all box and room lights were extinguished to preclude any possible contribution of visual information to the judgments of body orientation. The procedure for each trial was the same as for the illuminated condition, except that there was no instruction to open the eyes and there was an additional delay of $1 \mathrm{sec}$ when the instructions to open the eyes would have been given.

\section{Experimental Design}

The independent variables were:

Box pitch. Pitch backward $20^{\circ}$, level, pitch forward $20^{\circ}$ (Figure 3).

Optic structure. Grid pattern, parallel luminous lines, darkness. Goal orientation. Erect, $45^{\circ}$ back from erect (Figure 2).

Initial bed pitch. $15^{\circ}$ back from goal orientation, goal orientation, $15^{\circ}$ forward from goal orientation.

Behavior in the darkness condition was used as a baseline against which to compare behavior in the illuminated conditions. Pitching the box in darkness enabled control for influences on spatial orientation that might result from artifactual auditory cues (e.g., from the sounds of positioning the bed and the box) or visual cues (e.g., from stray light sources). The level box pitch condition, in illumination and in darkness, allowed independent evaluation of the in fluence of forward versus backward box pitch on body orientation; similarly, the initial bed-at-goal condition allowed independent evaluations of the influence of forward versus backward bed pitch on body orientation. Thus, the darkness condition, the level box pitch condition, and the initial bed-at-goal condition can be considered as controls.

In a within-subject, full factorial design, the order of presentation of optic structure was counterbalanced across subjects; within each level of optic structure, the orders of box pitch, goal orienta-
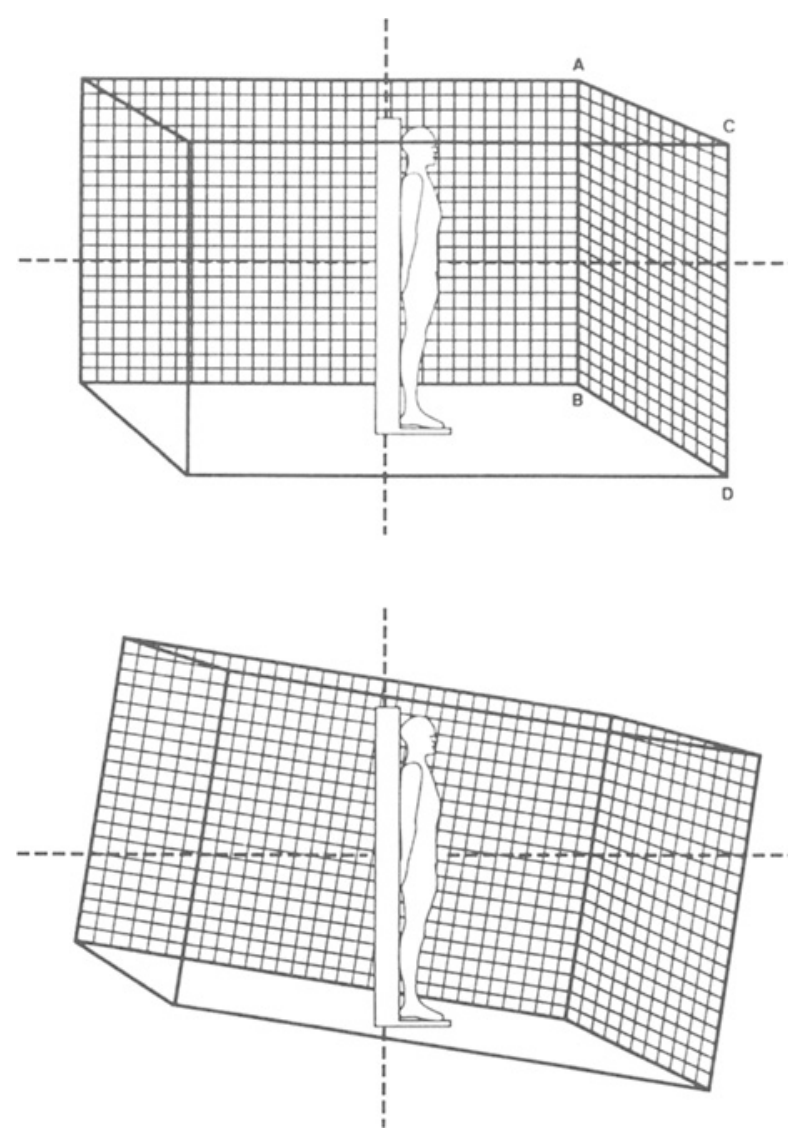

Figure 3. Pitch box in level (top) and box forward (bottom) conditions with the subject erect (for simplicity, the box backward condition is not illustrated here). 
tion, and initial bed pitch were randomly presented without replacement to yield 18 trials for each level of optic structure. There were 54 trials in each experimental session. The dependent variable was the signed deviation of body position from the desired goal, in degrees.

Because some previous investigations of the influences of tilted optic environments on spatial perception and orientation revealed differences related to the gender of the subjects (Lichtenstein \& Saucer, 1974; Morf, Kavanaugh, \& McConville, 1971; Witkin et al., 1954), we initially performed a five-way, split-plot analysis of variance (ANOVA) with gender as the fifth variable. Our analysis revealed no significant main effects or interactions of gender on deviations of body orientation. Consequently, a four-way, repeated measures ANOVA, collapsed across gender, was performed.

Tests of the desired cell differences were accomplished with planned-comparison $t$ tests at the 0.01 significance level (Edwards, 1985). Comparisons of cell variances were accomplished with statistical tests suggested by Games (1978) and Levy (1975).

Negative deviations from the vertical or diagonal goal denote errors in the supine direction (pitch backward), and positive deviations denote errors in the prone direction (pitch forward).

\section{RESULTS}

\section{Box Pitch and Optic Structure}

Pitch of the box resulted in bias of postural orientation in the direction of the pitch $[F(2,22)=45.51, p<.001]$. When the box was level, the mean deviation resulting from attempts to set the body to the goal was $-2.1^{\circ}$. Initial backward box pitch resulted in a mean deviation of $-4.7^{\circ}$, and initial forward box pitch resulted in a $0.8^{\circ}$ deviation, with a range of $5.5^{\circ}$, yielding a bias ratio of $14 \%$.

There was no main effect for optic structure $[F(2,22)=$ $1.33, p=.29$ ], presumably because this factor included data collected under conditions of both forward and backward box pitch. However, the interaction of box pitch and optic structure revealed that the pitched grid was more effective at influencing body orientation than the pitched luminous lines and that the pitched lines were more effective at influencing body orientation than pitching the box in darkness $[F(4,44)=22.77, p<.001$; Figure 4$]$. In darkness, the effect of box pitch (obtained by linear

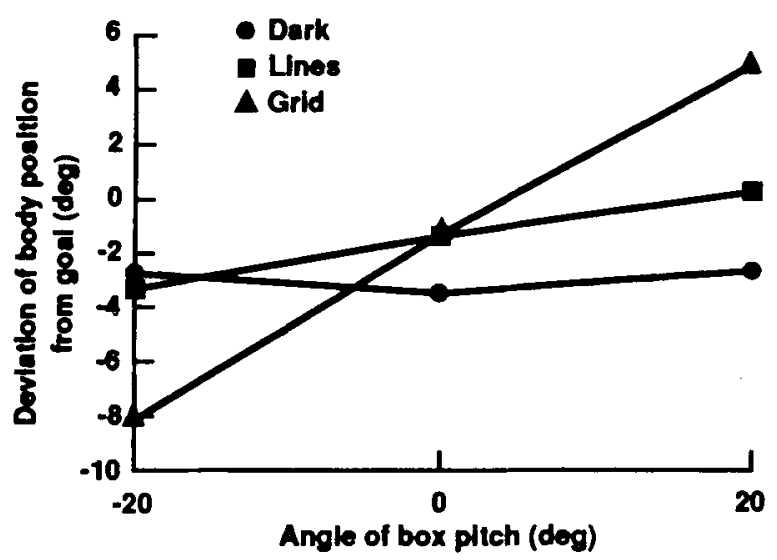

Figure 4. Effects of box pitch on perceived body orientation for the dark, luminous lines, and grid conditions.

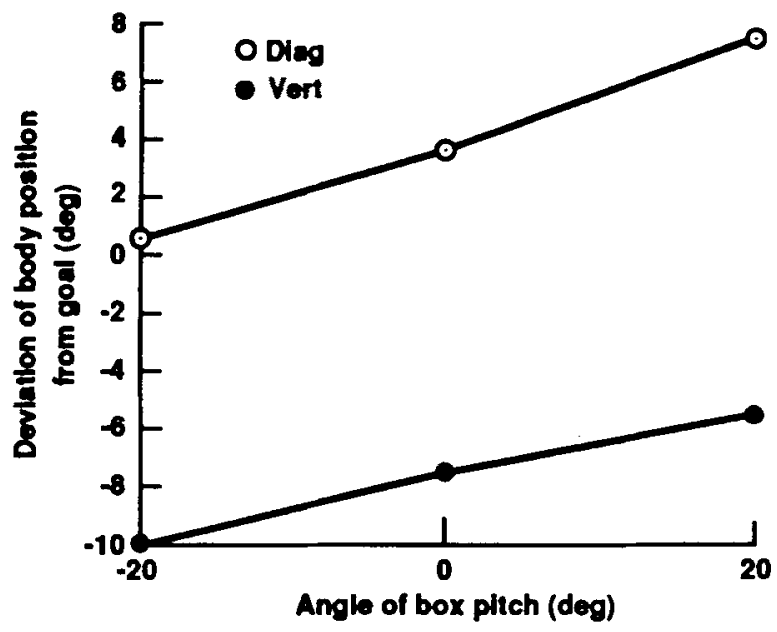

Figure 5. Effects of box pitch on perceived body orientation while the subjects attempted to move to the diagonal (Diag) and vertical (Vert) goals.

regression) was $-0.1^{\circ}$ for $\pm 20^{\circ}$ of box pitch ( $0 \%$ optic bias). This lack of an effect was expected because the box was not visible. With visual stimulation, the effect of box pitch on body orientation was significantly greater when the grid was present $\left(13^{\circ}\right.$, a $33 \%$ optic bias) than when only two parallel luminous strips were present $\left[3.5^{\circ}\right.$, a $9 \%$ optic bias; $t(44)=9.56, p<.001]$; the effect of the pitched luminous lines on body orientation was significantly greater than the effect of pitching the box while the subjects were kept in total darkness $[t(44)=3.52$, $p<.01]$.

\section{Box Pitch and Goal Orientation}

The subjects tended to orient forward from the diagonal goal and backward from the vertical goal, resulting in a statistically significant effect of goal orientation $[F(1,11)=$ $45.05, p<.001$; Figure 5].

Figure 5 demonstrates that the effect of box pitch was significantly greater when the goal was the diagonal position $\left(6.8^{\circ}\right.$, a $17 \%$ optic bias) than when the goal was the vertical position $\left[4.2^{\circ}\right.$, an $11 \%$ optic bias; $F(2,22)=$ $5.98, p<.01]$. Because this box pitch $\times$ goal orientation interaction includes data collected in the dark, the influence of the pitched box is attenuated in this two-way interaction.

\section{Box Pitch $\times$ Optic Structure $\times$ Goal Orientation}

The interaction of box pitch $\times$ optic structure $\times$ goal orientation was not statistically significant $[F(4,44)=$ $1.56, p=.2]$. Planned comparisons tested the effects of box pitch as a function of optic structure and goal orientation (Figure 6). There was 0\% optic bias in the dark for both the vertical and the diagonal goals. The pitched grid pattern biased postural orientation to the diagonal goal $\left(15.7^{\circ}\right.$, a $39 \%$ optic bias) more than to the vertical goal $\left[10.3^{\circ}\right.$, a $26 \%$ optic bias; $\left.t(44)=4.84, p<.01\right]$. The pitched luminous lines also biased orientation to the diagonal goal $\left(4.8^{\circ}\right.$, a $12 \%$ optic bias) more than to the ver- 


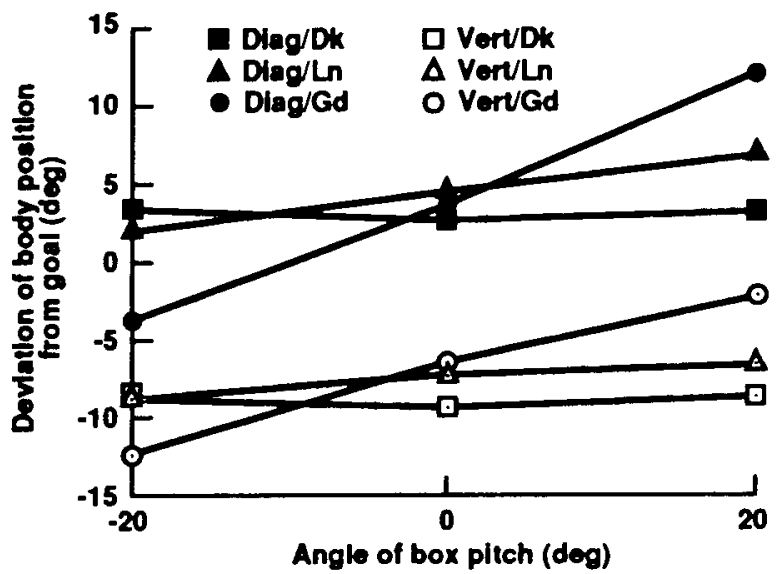

Figure 6. The interaction of box pitch, optic structure, and goal orientation. The pitched grid (Gd) and the pitched luminous lines (Ln) bias orientation to both the diagonal (Diag) and the vertical (Vert) goals; the magnitude of the bias is greater when the subjects orient to the diagonal than to the vertical goal. There was no influence of the pitched box in the dark (Dk) condition.

tical goal $\left[2.1^{\circ}\right.$, a $5 \%$ optic bias; $\left.t(44)=2.42, p<.05\right]$. The optic bias of orientation which resulted from pitching the luminous lines was greater than that obtained in darkness when the subjects oriented to the diagonal goal but not when they oriented to the vertical goal $[t(44)=$ $4.3, p<.01]$. These results indicate a nonadditive contribution of visual stimuli to body orientation and suggest that the influence of visual information depends upon the context provided by the distribution of somatosensory cues.

\section{Variability in Box Pitch $\times$ Optic Structure $\times$ Goal Orientation}

Examination of orienting behavior in control conditions, that is, those in which there was no initial box pitch or initial deviation of the bed from the goal, reveals how well the subjects perceived body position in the absence of the biasing influences of the deviated box or bed. Figure 7 depicts the constant errors ( $C E s$ ) and between-subject standard deviations $(S D s)$ of these control settings for each of the six optic structure $\times$ goal orientation conditions. Within each goal orientation, the $C E$ s were not statistically different from each other as a function of optic structure $[t(88)=0.23, p>.01]$; however, there were differences in response variability. ${ }^{3}$

When the subjects oriented to the diagonal goal, the $S D \mathrm{~s}$ obtained in darkness and in the luminous lines and grid conditions were not different from each other; when the subjects oriented to the vertical goal, the $S D$ obtained in darkness $\left(8.1^{\circ}\right)$ was greater than the $S D$ s in the luminous strip $\left(4.1^{\circ}\right)$ or the grid environment $\left[4.1^{\circ} ; d_{\text {crit }}(.01,3,33)=\right.$ 2.11]. In addition, for the darkness condition, the $S D$ of setting the body to the diagonal goal did not differ significantly from the $S D$ of setting the body to the vertical goal $\left[d_{\text {crit }}(.01,2,22)=2.0\right]$. In contrast, for the luminous lines and grid conditions, variability in setting the body to the diagonal goal was greater than that in setting the body to the vertical goal $\left[d_{\text {crit }}(.01,2,22)=2.0\right]$. Response variability in setting the body to these goals was not different when the box was pitched or when the bed was displaced from the goal.

\section{Initial Bed Pitch}

The perception of body position was biased by the initial starting position $[F(2,22)=42.15, p<.001]$. When the initial bed pitch was at the goal, the mean deviation resulting from attempts to set the body to the goal was $-2.4^{\circ}$. Initial backward bed pitch resulted in a mean deviation of $-5.1^{\circ}$, and initial forward bed pitch resulted in a $1.5^{\circ}$ deviation, with a range of $6.6^{\circ}$, yielding a bias ratio of $22 \%$. Bias of perceived body orientation in the direction of initial bed pitch may result from an underestimation of the extent of body pitch due to adaptation of somatosensory receptors.

\section{Initial Bed Pitch and Box Pitch}

The interaction of initial bed pitch and box pitch was not statistically significant $[F(4,44)=1.57, p=.2]$. The main effects for both box pitch and initial bed pitch in the absence of a significant interaction indicates that each of these variables independently influences perceived body orientation.

\section{Initial Bed Pitch $\times$ Box Pitch $\times$ Optic Structure}

The interaction of initial bed pitch $\times$ box pitch $\times$ optic structure was not significant $[F(8,88)=1.01, p=$ .44]. We were interested in determining whether starting position was influential in biasing postural orientation both in the light and in darkness. The clearest way to study this issue is to examine the condition in which the surrounding box is not pitched from the horizontal. Planned comparisons of starting position as a function of

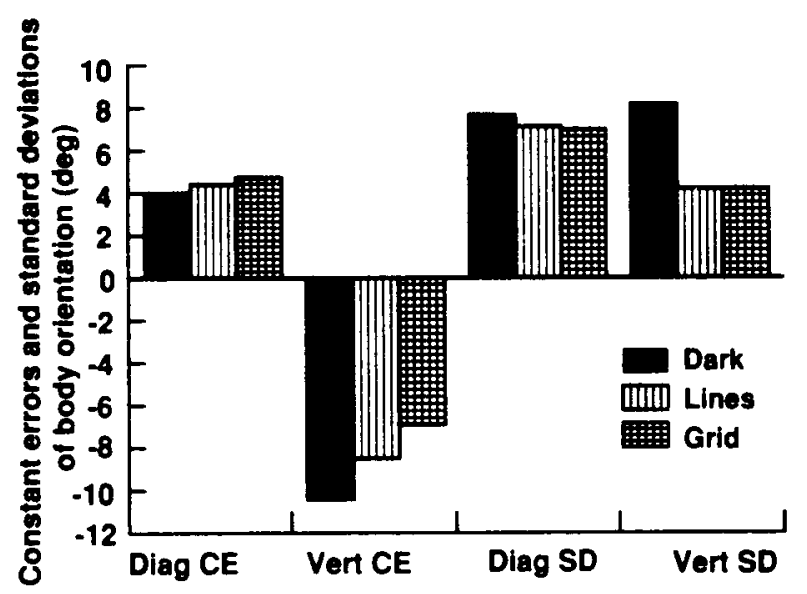

Figure 7. Constant errors (CEs) and standard deviations (SDs) of postural orientation for each of the six optic structure $\times$ goal orientation conditions. Only data from the control condition, the condition in which there was no initial box pitch or initial deviation of the bed from the goal, are shown. Diag and Vert indicate results obtained when orienting to the diagonal and vertical goals, respectively. 


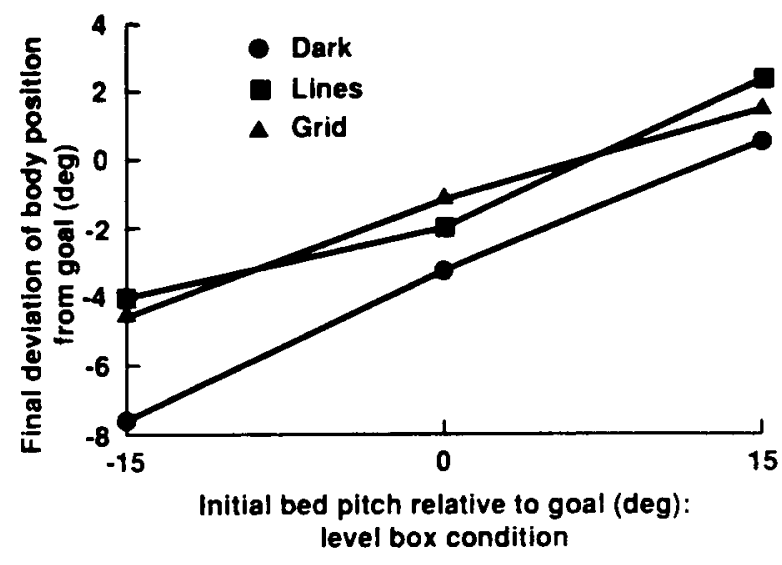

Figure 8. The influence of initial body position as a function of box pitch and optic structure.

optic structure in the level box condition also failed to indicate differences in the bias due to starting position between illuminated and darkness conditions (Figure 8). Thus, the effects of starting position appear to be independent of visual influence.

\section{Initial Bed Pitch and Goal Orientation}

The interaction of initial bed pitch and goal orientation was not statistically significant $[F(2,22)=0.5, p=.62]$. Significant main effects of both box pitch and goal orientation and no significant interaction indicates that these variables independently influence body orientation.

\section{DISCUSSION}

Our results demonstrate that body orientation is influenced by the pitch of the optic environment, the structure of the optic environment, the goal to which the subject attempts to set his/her body, and the initial orientation of the subject's body. When the box was pitched forward or backward, orientation settings were biased in the same direction as the box was pitched. The pitched grid pattern was shown to be most effective in biasing settings of body orientation, the pitched luminous lines were less effective, and the darkness condition produced no effects of box pitch. The effects of box pitch were greater when the subject attempted to orient to the diagonal than to the vertical goal. The initial pitch of the body, forward or backward from the goal, biased final settings of the body in the same direction.

Lateral tilting of a luminous frame results in perception of body tilt in the opposite direction of the frame tilt (Sigman, Goodenough, \& Flannagan, 1978). We assume that the pitched optic array in our experiment results in a similar misperception of body orientation in the median plane, and that the subjects compensate by moving in the direction of the pitched optic array in attempting to orient their bodies to a gravitational reference. The subsequent deviation occurs despite cognitive understanding that the orientation of the optic array is not necessarily congruent with the direction of gravity.

Emmert (1989), also using a pitched box to study postural orientation, obtained $0 \%$ optic bias when subjects oriented to the vertical goal, whereas we obtained $26 \%$ optic bias. It is possible that the reduced optic structure of her apparatus-she employed blank white walls instead of a dense grid pattern-may be partially responsible for the smaller influence of her pitched box.

The effects of pitched optic stimuli on spatial orientation have been observed in other settings. Matin et al. (1987) found that the biasing effect of a pair of pitched luminous lines on visually perceived eye level was not significantly different from the $61 \%$ optic bias that he obtained for subjects placed in a richly structured pitched environment (Matin \& Fox, 1989, 1990). In contrast, the present data show different effects of pitched luminous lines and a pitched grid on postural orientation. The difference in results from the two studies suggests that the available optic inputs may contribute differently to the mechanisms underlying perceived eye level and perceived body orientation and/or they may carry different information value for the two spatial indicators. Thus, our results do not necessarily conflict with those of Matin et al. (1987).

The small effect of luminous lines in our study may reflect a general insensitivity of body orientation to deviated luminous visual stimuli. For example, Sigman et al. (1979) also reported a 9\% optic bias on body orientation when a luminous frame was rotated about the subject's roll axis, whereas a $26 \%$ optic bias was obtained when the same subjects adjusted a rod to the vertical. These results are consistent with other data that suggest that visual judgments of the vertical and perceived postural orientation are subserved by different mechanisms (Mittelstaedt, 1986).

Emmert's (1989) failure to find biasing effects of the pitched box when subjects oriented to the erect position also may have resulted from providing the subjects with a rigid foot support, whereas we placed foam padding between the rigid foot support and the subject's feet. Emmert's results, together with our data and those of Nyborg (1971; in which a tilted visual frame biased orientation of a line to the vertical more when the tilted body was supported by a padded structure than when it was supported by a rigid structure), suggest that the salience of somesthetic information constrains the extent to which visual information about the direction of gravity can influence spatial orientation.

We can attempt to understand how visual cues are used in setting the body to the gravitational vertical by examining the interaction of box pitch $\times$ optic structure $\times$ goal orientation. We found that the biasing effect of the pitched optic array is smaller for the vertical than for the diagonal goal. We also found that in the visual control condition, the variability in setting the body to the vertical goal is smaller than that in setting the body to the diagonal goal. These combined results suggest a nonlinear process in which visual cues are less informative for the perception 
of postural orientation when somesthetic cues are unambiguous, such as when the body is in the upright position.

Although the pitch of the optic environment biased body orientation to both diagonal and vertical goals, the effect of box pitch was greater when the subject oriented to the diagonal than to the vertical goal. It would be tempting to explain this effect on the basis of differences in optic stimulation under the two conditions. For example, Ebenholtz (1977) has shown that increased retinal size of a frame in a rod-and-frame test results in increased bias in setting the rod to the vertical. However, in the present study, the effect of the pitched environment on body orientation was not due solely to retinal size; the subject's head was further from the far wall of the box when attempting to orient to the diagonal than to the vertical goal. Thus, although the retinal angle subtended by a given structural element of the optic array was smaller for the diagonal goal, the optic bias was greater. Clearly, an explanation based on differences in retinal size is not adequate to explain these results.

The smaller optic bias in orienting to the vertical goal than to the diagonal goal in our experiment may be the result of enhanced information from otolith organs, internal organs (Mittelstaedt, 1991; Mittelstaedt \& Fricke, 1988), or cutaneous and proprioceptive cues from the legs resulting from weight bearing. If some combination of vestibular and somesthetic cues contributes to enhanced information in the upright position, one would expect that settings of the body to the vertical goal in the dark would be less variable than settings to the diagonal goal in the dark. Our data do not support this expectation (Figure 7). Instead, they indicate that although somesthetic cues alone do not provide more precise information for orienting the body to the vertical than to the diagonal goal, they do interact with optic cues to provide more precise information for orienting to the vertical goal (Figure 7).

The findings of nonsignificant differences between response variability in orienting to the diagonal versus the vertical goal in the dark may be surprising if one believes that the otolith organs provide more precise information about the direction of gravity when the body is upright (Knight, 1958; Nelson, 1967, 1968), but data from Nelson's experiment actually support our findings. Nelson required normal blindfolded subjects to orient themselves under water; he demonstrated that vestibular cues, with greatly reduced or absent visual, proprioceptive, and cutaneous pressure cues, provide only very crude information about body orientation with respect to gravity. In Nelson's well-controlled study, his submerged subjects were able to orient themselves only to the major coordinate axes. In orienting to the upright position, the $S D$ was $15.2^{\circ}$, which was not significantly different from the $S D$ of $16.2^{\circ}$ obtained for orienting to the supine position. ${ }^{4}$ We can infer from Nelson's data that otolith cues are probably no more sensitive when the body is in the erect position than when it is in the $45^{\circ}$-back-from-erect position. In contrast, Nelson found that orienting to the upside-down position resulted in a significantly greater $S D$ of $33.8^{\circ}$.
It is not clear which sources of somesthetic information may interact with visual information to determine postural orientation. We can eliminate one potential source by examining the interactions of initial bed pitch $\times$ box pitch and initial bed pitch $\times$ goal orientation. The presence of main effects on body orientation for initial bed pitch, box pitch, and goal orientation, with no significant interactions of initial bed pitch with either box pitch or goal orientation, suggests an independent contribution of starting position to body orientation. Since starting position bias is probably due to adaptation of somesthetic cues (Clark \& Graybiel, 1963, 1964; Howard \& Templeton, 1966), and since a 10-sec delay at the starting position probably adapts cutaneous, but not proprioceptive, cues (Nemire, 1992), we presume that the adapted cutaneous information about body orientation is evaluated independently of visual, vestibular, and proprioceptive information to determine the perception of body position. We do not propose that nonadapted cutaneous spatial cues are evaluated independently of information provided by other sensory systems; however, we do not rule out the possibility.

The interaction of visual cues with somesthetic cues provided by the internal organs (Mittelstaedt, 1991; Mittelstaedt \& Fricke, 1988) may also play a role in postural orientation. Although difficult, it is not impossible to imagine how information about postural orientation provided by internal organs such as the kidneys (Mittelstaedt, 1991) may contribute to an internal spatial model of one's relation to gravity by combining with spatial information provided by retinal inputs.

Another way in which visual information may contribute to postural orientation is through interaction with vestibular and proprioceptive cues. For example, it is known that vestibulo-spinal reflexes contribute to the stabilization of postural sway (Allum \& Pfaltz, 1985; Mauritz \& Dietz, 1980). In the upright position, vestibulo-spinal mechanisms operate appropriately. In contrast, when the body is positioned around the diagonal goal $\left(30^{\circ}\right.$ to $60^{\circ}$ back from vertical), support of the body is shifted from the legs to the dorsal aspects of the body; it is possible that reducing the load on the leg muscles may circumvent the normal operation of the orienting reflexes, causing greater reliance on visual cues.

We speculate that the greater influence of the pitched optic array on body orientation when subjects attempt to orient to the diagonal as opposed to the vertical goal results from the interaction of visual, vestibular, and proprioceptive cues. Reduced proprioceptive information about postural orientation when the body is in the diagonal position results in larger effects of the pitched optic array. Adaptation of cutaneous cues when the body is in the diagonal position reduces the available information about body position, resulting in a larger influence of the pitched optic array on body orientation. The combined effect is an increase in the influence of the pitched optic array on postural orientation.

We further examined how visual, vestibular, and proprioceptive cues may act to influence body orientation by 
attempting to compare the variability of body settings obtained in the present experiment with those obtained by Emmert (1989) and Nelson $(1967,1968)$. Although these comparisons involve different experiments, numbers of subjects, measurements, and methods, which can compromise their validity, our findings are highly suggestive of a hierarchical model for postural orientation. In Emmert's study, subjects oriented to the upright position while their bodies were supported by a rigid surface. In the dark, the standard deviation was $2.5^{\circ}$, and in the light, when the surrounding box was level, the standard deviation was $2.7^{\circ}$ (Figure 9A). Adding visual information did not decrease variability; reliable somesthetic cues provided by a rigid surface of support were sufficient for performing the task.

In contrast, addition of veridical visual cues about the direction of gravity does reduce response variability when subjects stand on a foam cushion: in the current experiment, the standard deviation of body orientation was $4.1^{\circ}$ in the light and $8.1^{\circ}$ in the dark (Figures $9 \mathrm{~B}$ and $9 \mathrm{C}$, respectively). Reducing the proprioceptive and cutaneous cues to body orientation by providing a deformable surface of support results in even greater variability of orientation than that observed in Emmert's (1989) study. This result is consistent with arguments provided by Stoffregen and Riccio (1988).

Water-immersion further reduces the surface of support (Nelson, 1967, 1968; Stoffregen \& Riccio, 1988). When water-immersed subjects move their bodies to the upright position in the dark, only vestibular cues are available to provide reliable information about the orientation of the body with respect to gravity. In this situation, the precision of orientation is not good (Figure 9D), although

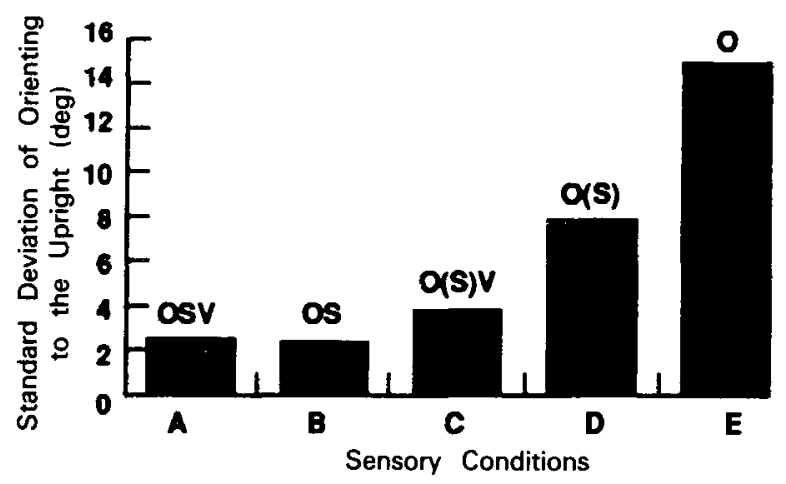

Figure 9. Hierarchical constraints on postural orientation. Standard deviations of adjustments to an erect postural orientation when the subject stands on a rigid support and orientation is accomplished in the dark, when only vestibular and somesthetic cues are available $\left(B, 2.5^{\circ}\right)$, and in the light, when visual cues are also available (A, 2.7 ${ }^{\circ}$; Emmert, 1989), (C) the subject stands on foam padding in the light $\left(4.1^{\circ}\right)$, (D) the subject stands on foam padding in the dark $\left(8.1^{\circ}\right)$, and (E) the subject wears a blindfold and is immersed in water so that vestibular cues are virtually the only cues available $\left(15.2^{\circ}\right.$; Nelson, 1967). " $O, " ~ " S$," and "V" refer to the availability of otolith, somesthetic, and visual cues, respectively; "(S)" refers to the reduction in the salience of the somesthetic cues because of the foam surface of support.
Evaluation Integration Exocution

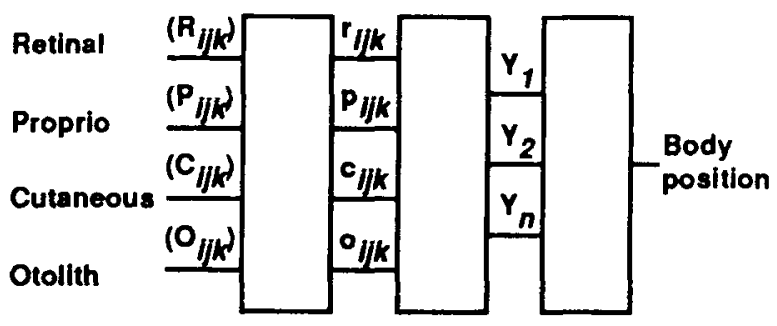

Figure 10. General model of spatial orientation (adapted from Massaro, 1987). We include only those sensory modalities and effectors that were manipulated in this experiment. A complete model would also include oculomotor, canal, and visceral inputs, specification of the intramodality influences ( $i j k)$, as well as outputs to the eyes, head, and arms. We do not intend to represent a serial-stage process; exclusion of possible feedback processes and temporal relationships are to keep the diagram simple for purposes of this paper. ("Proprio" = proprioceptive inputs.)

these cues do provide some constraints on orientation in that subjects were able to maintain orientation within the appropriate quadrant. Note that in this task, visceral cues to orientation are also available (Mittelstaedt, 1991; Mittelstaedt \& Fricke, 1988). The poor orientation observed in this situation suggests that even the combination of otolith and visceral information is only grossly adequate to orient the body to the upright position.

Comparisons of data in the present experiment with those from Nelson $(1967,1968)$ suggest that proprioceptive and cutaneous cues are more important for orienting the body to the upright than are otolith cues (Stoffregen \& Riccio, 1988). Note that in our study, the foam surface of support resulted in greater variability of body orientation and larger magnitude of optic bias by the pitched optic array than was observed in Emmert's (1989) study, which employed a rigid surface of support. Comparison of these data suggests that the rigidity of the surface of support influences the way in which visual information is used to orient the body to the upright.

The relationship of visual, vestibular, and somesthetic inputs in determining the perception of body orientation is consistent with models describing nonlinear interactions of visual and vestibular cues to spatial orientation as indicated by adjusting a rod to the apparent vertical (e.g., Bischoff, 1974; Schone \& Udo de Haes, 1971; Young, 1984), an exocentric task. We extend the scope of these models by providing data that the contribution of visual cues to postural orientation, an egocentric task, also varies as a nonlinear function of the vestibular and somesthetic cues.

We have adapted a general model of sensory integration based on "modality precision" models of intersensory interaction (reviewed in Welch \& Warren, 1986) and a general model of intersensory interaction postulating a combination of multiple ambiguous sensory inputs to perception (Brunswik, 1956; Massaro, 1987). Our model (Figure 10) assumes mechanisms by which multiple ambiguous sources of sensory inputs describing body orientation are combined to reduce the probability of error in correctly orienting the body to gravitational coordinates. 
There are three stages involved in this model; they are evaluation, integration, and execution. In the evaluation stage, information about body orientation with respect to gravity is extracted from each of the relevant sensory inputs $\left(R_{i j k}, P_{i j k}\right.$, etc. $)$ and transformed into a sensory spatial code $\left(r_{i j k}, p_{i j k}\right.$, etc. $)$ indicating the spatial location of the body with respect to external space. In a given situation, some sensory inputs are more informative than others. For example, visual stimulation provided by the grid pattern in our experiment is more informative than the stimulation provided by the pair of luminous lines. In the integration stage, the transformed spatial codes from the multiple inputs are combined to form perceptual codes $\left(Y_{n}\right)$. Integration is based on both tonic and phasic information states and on hierarchical constraints resulting from the variability of the sensory stimulation (Figure 9). In this stage, for example, visual information is combined with somesthetic cues to determine spatial orientation. Because adapted cutaneous cues independently contributed to postural orientation, we postulate the existence of multiple outputs from the integration stage $\left(Y_{1}, Y_{2}, \ldots Y_{n}\right)$. In the execution stage, the integrated sensory information is mapped onto the motor spatial system so that the body may be oriented appropriately.

Knowledge of intersensory interactions that contribute to body orientation in terrestrial environments can contribute to our understanding of perception and orientation in other environments where normal sensory relations are altered. Such environments include those encountered in aircraft and spacecraft, as well as hostile conditions that require remotely operated equipment. For example, the misperception of the pitch of a flying aircraft relative to the horizontal axis (Cohen, 1977; Gilson, Guedry, Hixson, \& Niven, 1973) and the misperception of the pitch of a visually presented landing strip (Kraft, 1978; Perrone, 1984) have been strongly implicated in several aviation accidents. In addition, examination of visual and somesthetic cues that bias postural orientation may lead to countermeasures for the spatial disorientation observed during orbital flight (Kerwin, 1975).

\section{REFERENCES}

Allum, J. H. J., \& Pfaltz, C. R. (1985). Visual and vestibular contributions to pitch sway stabilization in the ankle muscles of normals and patients with bilateral vestibular deficits. Experimental Brain Research, 58, 82-94.

Andersen, G. J., \& Braunstein, M. L. (1985). Induced self-motion in central vision. Journal of Experimental Psychology: Human Perception \& Performance, 11, 122-132.

Asch, S. E., \& Wirkin, H. A. (1948a). Studies in space orientation: I. Perception of the upright with displaced visual fields. Journal of Experimental Psychology, 38, 325-337.

Asch, S. E., \& WITkIN, H. A. (1948b). Studies in space orientation: II. Perception of the upright with displaced visual fields and with body tilted. Journal of Experimental Psychology, 38, 455-477.

Bischoff, N. (1974). Optic-vestibular orientation to the vertical. In H. H. Kornhuber (Ed.), Handbook of sensory physiology: Vol. 6. Vestibular system (Pt. 2, pp. 155-190). New York: SpringerVerlag.

Brunswik, E. (1956). Perception and the representative design of psychological experiments. Berkeley: University of California Press.
Clark, B., \& Graybiel, A. (1963). Perception of the postural vertical in normals and subjects with labyrinthine defects. Journal of $E x$ perimental Psychology, 65, 490-494.

Clark, B., \& Graybiel, A. (1964). Perception of the postural vertical following prolonged bodily tilt in normals and subjects with labyrinthine defects. Acta Oto-laryngologica, 58, 143-148.

Clark, F. J., \& Horch, K. W. (1986). Kinesthesia. In K. R. Boff, L. Kaufman, \& J. P. Thomas (Eds.), Handbook of perception and human performance: Vol. I. Sensory processes and perception (pp. 13-1-13-62). New York: Wiley.

COHEN, M. M. (1977). Disorienting effects of aircraft catapult launchings: III. Cockpit displays and pilot performance. Aviation, Space. \& Environmental Medicine, 48, 797-844

COHEN, M. M., \& LARSON, C. A. (1974). Human spatial orientation in the pitch dimension. Perception \& Psychophysics, 16, 508-512.

Collier, R. O., Jr., Baker, F. B., Mandeville, G. K., Hayes, T. F. (1967). Estimates of test size for several test procedures based on conventional variance ratios in the repeated measures design. Psychometrika, 32, 339-353.

EBENHOLTZ, S. M. (1977). Determinants of the rod and frame effect: The role of retinal size. Perception \& Psychophysics, 22, 531-538.

Ebenholtz, S. M., \& Benzschawel, T. L. (1977). The rod and frame effect and induced head tilt as a function of observation distance. Perception \& Psychophysics, 22, 491-496.

EDWARDS, A. L. (1985). Experimental design and psychological research, (5th ed.). New York: Harper \& Row.

EMMERT, L. T. A. (1989). The effects of visual and proprioceptive inputs on human spatial orientation in the pitch dimension. Unpublished master's thesis, Naval Postgraduate School, Monterey, CA.

Games, P. A. (1978). A three-factor model encompassing many possible statistical tests on independent groups. Psychological Bulletin, 85. 168-182

Geisser, S., \& Greenhouse, S. W. (1958). An extension of Box's results on the use of the $F$ distribution in multivariate analysis. Annals of Mathematical Statistics, 29, 885-891.

GibSON, J. J. (1961). Ecological optics. Vision Research, 1, 253-262.

Gilson, R. D., Guedry, F. E., Jr., Hixson, W. C., \&iven, J. I. (1973). Observations on perceived changes in aircraft attitude attending head movements made in a 2-g bank and turn. Aerospace Medicine, 44, 90-92.

Goodenough, D. R., Oltman, P. K., Sigman, E., Cox, P. W. (1981). The rod-and-frame illusion in erect and supine observers. Perception \& Psychophysics, 29, 365-370.

Greenhouse, S. W., \& Geisser, S. (1959). On methods in the analysis of profile data. Psychometrika, 24, 95-112.

HAy, J. C., PiCK, H. L., JR., IKedA, K. (1965). Visual capture produced by prism spectacles. Psychonomic Science, 2, 215-216.

HowARD, I. P. (1982). Human visual orientation. Chichester: Wiley.

HowARD, I. P., Templeton, W. B. (1966). Human spatial orientation. London: Wiley.

KEPPEL, G. (1973). Design and analysis: A researcher's handbook. Englewood Cliffs, NJ: Prentice-Hall.

Kerwin, J. P. (1975). Weightlessness: A case history. Acta Astronautica, 2, 85-87.

KNIGHT, L. A. (1958). An approach to the physiologic simulation of the null-gravity state. Journal of Aviation Medicine, 29, 283-286.

Kraft, C. L. (1978). A psychophysical contribution to air safety: Simulator studies of visual illusions in night visual approaches. In $\mathrm{H}$. A. Pick, H. W. Leibowitz, J. R. Singer, A. Steinschneider, \& H. W. Stevenson (Eds.), Psychology: From research to practice (pp. 363385). New York: Plenum.

LeChNER-STEINLEITNER, S., \& SCHONe, H. (1980). The subjective vertical under 'dry' and 'wet' conditions at clockwise and counterclockwise changed positions and the effect of a parallel-lined background field. Psychological Research, 41, 305-317.

LEE, D. N., \& ARONSON, E. (1974). Visual proprioceptive control of standing in human infants. Perception \& Psychophysics, 15, 529-532.

LEVY, K. J. (1975). Comparing the variances of several treatments with a control. Educational \& Psychological Measurement, 35, 793-796.

Lichtenstein, J. H., SAUCER, R. T. (1974). Visual dependency in the erect and supine positions. Journal of Applied Psychology, 59, 529-531. 
MASSARO, D. W. (1987). Speech perception by eye and ear: A paradigm for psychological inquiry. Hillsdale, NJ: Erlbaum.

MAtIN, L. (1986). Visual localization and eye movements. In K. R. Boff, L. Kaufman, \& J. P. Thomas (Eds.), Handbook of perception and human performance: Vol. 1. Sensory processes and perception (pp. 20-1-20-45). New York: Wiley.

Matin, L., \& Fox, C. R. (1989). Visually perceived eye level and perceived elevation of objects: Linearly additive influences from visual field pitch and from gravity. Vision Research, 29, 315-324.

MatiN, L., \& Fox, C. R. (1990). Visually perceived eye level and perceived elevation of objects: Linearly additive influences from visual field pitch and from gravity (corrigenda). Vision Research, 30, 1.

Matin, L., Fox, C. R., Doktorsky, Y. (1987). How high is up? Investigative Ophthalmology \& Visual Sciences, 28(Suppl.), 300.

MATIN, L., \& LI, W. (1989). A single pitched line in darkness controls elevation of visually perceived eye level. Investigative Ophthalmology \& Visual Sciences, 30(Suppl.), 506.

MATIN, L., \& LI, W. (1992). Visually perceived eye level: Changes induced by a pitched-from-vertical 2-line visual field. Journal of Experimental Psychology: Human Perception \& Performance, 18, 257-289.

Mauritz, K.-H., \& DietZ, V. (1980). Characteristics of postural instability induced by ischemic blocking of leg afferents. Experimental Brain Research, 38, 117-119.

MitTELSTAEDT, H. (1983). Subjective vertical in weightlessness. In M. Igarashi \& F. O. Black (Eds.), Vestibular and visual control on posture and locomotor equilibrium. Seventh International Symposium of the International Society of Posturography (pp. 139-150). Basel: Karger.

MrTtelSTAEDT, H. (1986). The subjective vertical as a function of visual and extraretinal cues. Acta Psychologica, 63, 63-85.

MITTELSTAEDT, H. (1991, July). Somatic versus vestibular gravity reception in man. Paper presented at the Sensing and Controlling Motion: Vestibular and Sensorimotor Function Conference of the New York Academy of Sciences, Palo Alto, CA.

Mittelstaedt, H., Fricke, E. (1988). The relative effect of saccular and somatosensory information on spatial perception and control. Advances in Oto-Rhino-Laryngology, 42, 24-30.

Morf, M. E., Kavanaugh, R. D., McConville, M. (1971). Intratest and sex differences on a portable rod-and-frame test. Perceptual \& Motor Skills, 32, 727-733.

NASHNER, L. M. (1971). A model describing visual detection of body sway motion. Acta Oto-laryngolica, 72, 429-436.

Nelson, J. G. (1967). The effect of water immersion and body position upon perception of the gravitational vertical (MF022.03.02-7002, Report No. 10, NADC-MR-6709). Warminster, PA: Naval Air and Development Center.

Nelson, J. G. (1968). Effects of water immersion and body position upon perception of the gravitational vertical. Aerospace Medicine, 39, 806-811.

Nemire, K. (1992). [Different adaptation rates for cutaneous and proprioceptive cues to postural orientation]. Unpublished data.

NYBORG, H. (1971). Tactile stimulation and perception of the vertical: I. Effects of diffuse vs. specific tactile stimulation. Scandinavian Journal of Psychology, 12, 1-13.

PASSEY, G. E. (1950). The perception of the vertical: IV. Adjustment to the vertical with normal and tilted visual frames of reference. Joumal of Experimental Psychology, 40, 738-745.

Perrone, J. A. (1984). Visual slant misperception and the 'black-hole' landing situation. Aviation, Space, \& Environmental Medicine, 55 , 1020-1025.

SCHONE, H., \& UDO DE HAES, H. A. (1971). Space orientation in humans with special reference to the interaction of vestibular, somaesthetic and visual inputs. Biokybernetic, 3, 172-191.

Sigman, E., Goodenough, D. R., \& Fannagan, M. (1978). Subjective estimates of body tilt and the rod-and-frame test. Perceptual \& Motor Skills, 47, 1051-1056.

Sigman, E., Goodenough, D. R., \& Flannagan, M. (1979). Instru- tions, illusory self-tilt and the rod-and-frame test. Quarterly Journal of Psychology, 31, 155-165.

Singer, G., Purcell, A. T., \& Austin, M. (1970). The effect of structure and degree of tilt on the tilted room illusion. Perception \& Psychophysics, 7, 250-252.

STOFFrEgen, T. A. (1986). The role of optical velocity in the control of stance. Perception \& Psychophysics, 39, 355-360.

Stoffregen, T. A., \& Riccio, G. E. (1988). An ecological theory of orientation and the vestibular system. Psychological Review, 95, 3-14.

Stoper, A. E., \& CoHen, M. M. (1989). Effect of structured visual environments on apparent eye level. Perception \& Psychophysics, 46, 469-475.

Streibel, M. J., Barnes, R. D., Julness, G. D., \& Ebenholtz, S. M. (1980). Determinants of the rod-and-frame effect: Role of organization and subjective contour. Perception \& Psychophysics, 27, $136-140$.

Templeton, W. B. (1973). The role of gravitational cues in the judgment of visual orientation. Perception \& Psychophysics, 14, 451-457.

WELCH, R. B., \& WARREN, D. H. (1980). Immediate perceptual response to intersensory discrepancy. Psychological Bulletin, 88, 638-667.

WELCH, R. B., \& WARREN, D. H. (1986). Intersensory interactions. In K. R. Boff, L. Kaufman, \& J. P. Thomas (Eds.), Handbook of perception and human performance: Vol. 1. Sensory processes and perception (pp. 25-1-25-36). New York: Wiley.

WITkIN, H. (1949). Perception of body position and of the position of the visual field. Psychological Monographs, 63, 1-46.

Witkin, H., Lewis, H. B., Hertzman, M., Machover, K., MeissNER, P. B., \& WAPNER, S. (1954). Personality through perception. New York: Harper.

YounG, L. R. (1984). Perception of the body in space: Mechanisms. In S. R. Geiger (Ed.), Handbook of physiology: Section I. The nervous system: Vol. 3. Sensory processes (Pt. 2, pp. 1023-1066). Bethesda: American Physiological Society.

Young, L. R., Oman, C. M., \& Dichgans, J. M. (1975). Influence of head orientation on visually induced pitch and roll sensation. Aviation, Space, \& Environmental Medicine, 46, 264-268.

\section{NOTES}

1. After Clark and Horch (1986, p. 13-2), we use the term "proprioception" to refer to sensations of "position, and muscle tension arising from muscles and associated structures." In addition, we use both "somesthetic" and "somatosensory" to refer to the combination of proprioceptive and cutaneous cues.

2. After Howard (1982, pp. 6-7), we refer to deviations in the midfrontal (coronal) plane as "roll" or "tilt" and deviations in the midsagittal (median) plane as "pitch" or "slant."

3 . We are aware that moderate heterogeneity of variance and of covariance in the ANOVA does not result in a seriously biased $F$ if there is an equal number of data points in each level of each independent variable (Collier, Baker, Mandeville, \& Hayes, 1967; Keppel, 1973). Nevertheless, we performed a conservative correction to the $F$ ratio (Geisser \& Greenhouse, 1958; Greenhouse \& Geisser, 1959). The correction resulted in an evaluation of $F$ at $d f=1,11$ for all effects in this design, yielding a critical value of $F \alpha=.05=4.84$. Only one of the effects that previously achieved significance, box pitch $\times$ goal orientation $\times$ initial bed pitch, failed to achieve significance at the $F$-corrected criterion. We eliminated further discussion of this interaction until further investigation.

4. The measure of variability presented in Nelson (1968) was the "elliptical probable error." To better compare Nelson's results with ours, we used between-subject SDs presented in Nelson (1967, Table I), after converting the units from radians to degrees.

(Manuscript received August 26, 1991; revision accepted for publication July 2, 1992.) 